\section{DIFFERENTIAL SENSITIVITY OF THORACIC MALIGNANT TUMORS TO ADENOVIRUS- MEDIATED DRUG SENSITIZATION GENE THERAPY}

Malignant mesothelioma may prove to be an attractive candidate for somatic gene therapy with replication-deficient recombinant adenovirus transfer of a toxic, or drug sensitization gene. Transfer of the herpes simplex thymidine kinase type I gene (HSVtk), followed by exposure to the acyclic nucleoside drug ganciclovir, has been shown to be an effective tumor cell killing system. To study generalized applicability, we tested a number of thoracic malignant cell lines for their sensitivity to gancyclovir after infection with an adenoviral vector containing the HSVtk gene (Ad.RSVtk). Using the concentration of gancyclovir required to kill $50 \%$ of the cells (IC50) as a measure of sensitivity, we detected variable sensitivity among cell lines, with mesothelioma most sensitive (IC50 $=0.075$ to $2.8 \mu \mathrm{mol} / \mathrm{L}$ gancyclovir), and non-small-cell carcinoma lines having an intermediate sensitivity (IC50 $=1.5$ to 100 $\mu \mathrm{mol} / \mathrm{L}$ ). In contrast, an ovarian carcinoma line was extremely resistant (IC50 > $2000 \mu \mathrm{mol} / \mathrm{L}$ ). To study the possible mechanisms for these differences, we studied cell lines with regard to their ability to be infected with an adenoviral vector containing a marker gene (Ad.CMVlacZ) and expression of the vitronectin receptor $\alpha_{v}$ (an integrin cell adhesion molecule shown to be required for adenovirus internalization after initial binding). We found that the degree of lac $Z$ transduction correlated with HSVtk sensitivity, whereas vitronectin receptor expression did not, suggesting that differences in initial viral binding ability, rather than internalization, may explain the sensitivity differences seen in vitro. (J THORAC CARDIOvasC SURG 1995;109:626-31)

W. Roy Smythe, $\mathrm{MD}^{\mathrm{a}}$ (by invitation), Harry C. Hwang, $\mathrm{MD}^{\mathrm{b}}$ (by invitation), Ashraf A. Elshami, $\mathrm{MD}^{\mathrm{b}}$ (by invitation), Kunjlata M. Amin, $\mathrm{PhD}^{\mathrm{a}}$ (by invitation), Steven M. Albelda, $\mathrm{MD}^{\mathrm{b}}$ (by invitation), and Larry R. Kaiser, MD, ${ }^{\mathrm{a}}$ Philadelphia, Pa.
$\mathrm{M}$ alignant pleural mesothelioma continues to be a clinically frustrating problem because of poor response to conventional medical and surgical therapies. Reported median survival is only 10 to 18 months and early local tumor recurrence is the rule. ${ }^{1,2}$ In addition to the fact that no effective conventional therapy for mesothelioma has been developed, the lack of a significant metastatic component and growth in the potential space of the

From the Department of Surgery, Section of General Thoracic Surgery, ${ }^{a}$ and the Department of Medicine, Section of Pulmonary and Critical Care, ${ }^{b}$ The Thoracic Oncology Research Laboratory, University of Pennsylvania Medical Center, Philadelphia, $\mathrm{Pa}$.

Read at the Seventy-fourth Annual Meeting of The American Association for Thoracic Surgery, New York, N.Y., April 24-27, 1994.

Address for reprints: Larry R. Kaiser, MD, The Thoracic Oncology Research Laboratory, Hospital of the University of Pennsylvania, 809 Maloney Bldg., 3600 Spruce St., Philadelphia, PA 19104.

Copyright $(\mathcal{C} 1995$ by Mosby-Year Book, Inc.

$0022-5223 / 95 \$ 3.00+0 \quad \mathbf{1 2 / 6 / 6 2 0 4 2}$ thoracic cavity make it an attractive candidate for somatic gene therapy. We have recently shown that recombinant replication-deficient adenovirus may be used to transfer novel genetic material to human mesothelioma both in vitro and in an in vivo xenograft tumor model $^{3}$ and that human mesothelioma cells are killed in vitro when the type $I$ herpes simplex thymidine kinase (HSVtk) gene is transferred via adenovirus followed by ganciclovir (GCV) exposure. ${ }^{4} \mathrm{GCV}$ is transformed by the viral thymidine kinase to GCV-triphosphate-a purine analog competing with normal cell nucleotides for deoxyribonucleic acid incorporation during replication and other processes. ${ }^{5}$ Surprisingly, uninfected tumor cells may be killed when infected cells expressing the HSVtk gene are treated with GCV (bystander effect). This phenomenon is thought to involve transfer of GCV-triphosphate from infected to uninfected cells via gap junctions or apoptotic vesicles. ., $^{7}$

One issue raised by these studies is the applicability of this approach to other cell types. For successful adenovirus-mediated gene transfer to occur, a number of steps must take place (Fig. 1), 


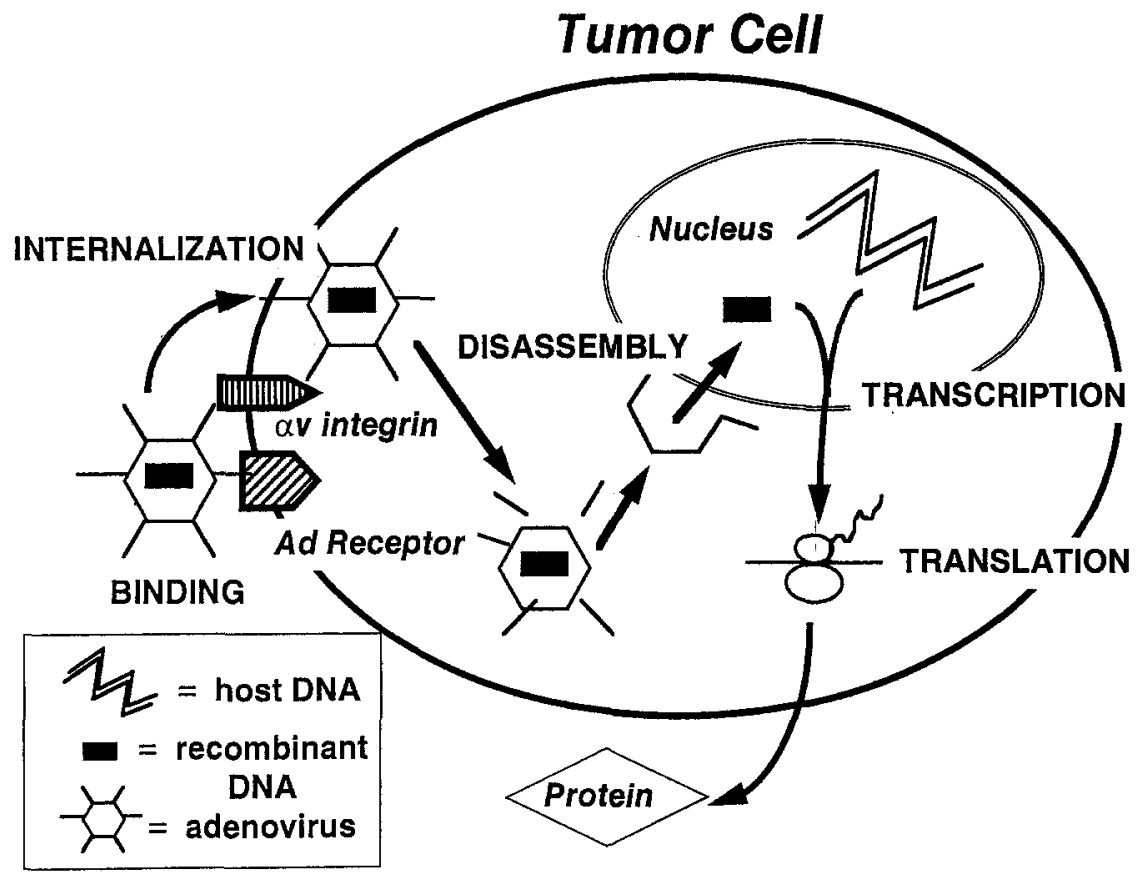

Fig. 1. Recombinant replication-deficient adenovirus gene transfer. The basic sequence of steps involved in adenovirus gene transfer leading to novel protein production in human tumor cells is schematically diagrammed. $D N A$, Deoxyribonucleic acid.

including initial viral binding (to an, as yet, unidentified cell surface receptor), internalization (a step dependent on the integrins $\alpha_{\mathrm{v}} \beta_{3}$ or $\alpha_{\mathrm{v}} \beta_{5}$ ), stepwise disassembly of viral proteins within endosomes, and transport of the recombinant genetic material to the nucleus where it may be transcribed and translated by the enzymatic machinery of the cell. ${ }^{8,9}$ Because differences in many of these mechanisms could be operative in various cell types, we tested the ability of Ad.RSVtk/GCV to induce cell killing in a number of mesothelioma and non-small-cell carcinoma lines and correlated sensitivity to GCV with the ability to express an adenovirus-transmitted marker gene and with vitronection receptor expression.

\section{Materials and methods}

Cell lines. Isolation and maintenance of the human mesothelioma lines REN, HeMe-B, 1-45, and non-smallcell carcinoma A549 are described elsewhere. ${ }^{3}$ The HPM normal human mesothelial cell line was the gift of Dr. C. Broaddas (University of California at San Francisco). Human non-small-cell carcinoma lines H460 and H1264 were the gift of Dr. C. Allegra (National Cancer Institute), and SK-MES-1, Calu-6 were obtained from ATCC (Rockville, Md). Ovcar-5 (human ovarian carcinoma) was the gift of Dr. T. Hamilton (Fox Chase Cancer Institute), and II-45 (rat mesothelioma) was furnished by Dr. C. Walker (M.D. Anderson Cancer Center). Cells were passaged in
RPMI (Mediatech, Washington, D.C.) with $10 \%$ fetal bovine serum (RPMI-10).

Recombinant adenovirus. The production of our adenovirus type 5 mutant replication-deficient vectors by homologous recombination techniques has been described in detail elsewhere. ${ }^{10,11}$ The Ad.CMVlacZ and Ad.RSV $t k$ constructs were the gift of Dr. J. M. Wilson (University of Pennsylvania Institute for Human Gene Therapy).

Cell culture infection with Ad.CMVlacZ and Ad.RSVtk. One hundred microliters of Ad.CMVlacZ (100 viral particles per tumor cell) was placed on confluent $2.5 \mathrm{~cm}^{2}$ per well culture plates. Plates were incubated at $37^{\circ} \mathrm{C}$ for 3 hours, and then virus was removed. For Ad.RSVtk experiments, confluent cultures of all lines in $25 \mathrm{~cm}^{2}$ flasks were infected with virus at a ratio of 100 particles per cell. One milliliter of viral solution was placed on the cells, which were then incubated at $37^{\circ} \mathrm{C}$ for 3 hours, after which time virus was removed.

$\mathrm{X}$-gal staining for $\boldsymbol{\beta}$-galactosidase (lac $Z$ gene transfer). Two days after infection with Ad.CMVlacZ, cells were stained with the $\beta$-galactosidase ( $\beta$-gal) substrate, $X$-gal. This staining method has been described in detail elsewhere. ${ }^{12}$ Expression of the gene for $\beta$-gal was indicated by discrete cells staining an intense blue color.

In vitro ganciclovir sensitivity assay. Viable cell number was assessed by means of a colorimetric assay (MTT cell proliferation assay, Promega Corporation, Madison, Wis.). Confluent $25 \mathrm{~cm}^{2}$ culture flasks were exposed to Ad.RSVtk for 3 hours (100 particles per cell). Twenty-four hours later, Ad.RSVtk-infected and uninfected cells were plated at 6000 cells per well in 96-well culture plates. After 
Table I. Comparison of in vitro sensitivity of a number of human thoracic tumor and other cell lines to the adenovirus vector-mediated $\mathrm{HSV} t \mathrm{k} / \mathrm{GCV}$ system, efficiency of transduction with an adenovirus transferring a marker gene (Ad.CMVlacZ), and percentage of cells expressing the $\alpha_{v}$ integrin by fluorescence activated cell sorting

\begin{tabular}{lccc}
\hline \multicolumn{1}{c}{ Cell line } & IC50 & lacZ+ & $\alpha_{v}+$ \\
\hline $1-45^{\mathrm{a}}$ & 0.075 & $90 \%$ & $53.7 \%$ \\
Calu-6 $^{\mathrm{b}}$ & 1.0 & $80 \%$ & $98.6 \%$ \\
SK-Mes-1 $^{\mathrm{b}}$ & 1.2 & $60 \%$ & $34.6 \%$ \\
REN $^{\mathrm{a}}$ & 1.5 & $60 \%$ & $46.7 \%$ \\
$\mathrm{HeMe} \mathrm{B}^{\mathrm{a}}$ & 2.8 & $20 \%$ & $\mathrm{~N} / \mathrm{A}$ \\
H460 & 10 & $30 \%$ & $65.5 \%$ \\
HPM & 25 & $5 \%$ & $8.4 \%$ \\
$\mathrm{~A} 549^{\mathrm{b}}$ & 75 & $5 \%$ & $98.4 \%$ \\
H1264 & 100 & $30 \%$ & $30.1 \%$ \\
II-45 (rat) & 60 & $30 \%$ & $\mathrm{~N} / \mathrm{A}$ \\
Ovcar5 $^{\mathrm{b}}$ & $>2000$ & $<1 \%$ & $53.7 \%$ \\
\hline
\end{tabular}

IC50, Concentration of GCV required to kill $50 \%$ of cells as compared with uninfected control cells of the same line; lacZ, the Escherichia coli $\beta$-galactosidase gene; $\alpha_{\mathrm{v}}$, cell surface integrin; cell line $e^{a}$, human malignant mesothelioma; cell lines ${ }^{b}$, human non-small-cell carcinoma; HPM, human normal mesothelial line; Ovcar5, human ovarian carcinoma; $I I-45$, Fisher rat malignant mesothelioma; $N / A$, not evaluated.

24 hours, medium was replaced with RPMI-10 containing 0 to $2000 \mu \mathrm{mol} / \mathrm{L}$ GCV, 9-[1,3 dihydroxy-2-propoxymethyl]-guanine (Syntex Laboratories, Palo Alto, Calif.). Five days later, cells were assayed with the MTT assay.

Fluorescence activated cell sorting for $\alpha_{\mathrm{v}}$ integrin. Cells were incubated with a negative control monoclonal antibody or $10 \mathrm{mg} / \mathrm{ml}$ of monoclonal human $\alpha_{\mathrm{v}}$ integrin antibody (gift of Dr. David Cheresh ${ }^{13}$ ) for 1 hour, and then incubated for 30 minutes with fluorescein isothiocyanate-conjugated $\mathrm{F}\left(\mathrm{ab}^{\prime}\right)^{2}$ goat antimouse immunoglobulin $G$. Cells from each tumor line were analyzed for fluorescence intensity by flow cytometry with a Cytofluorograph $50 \mathrm{H}$ sorter equipped with 2150 Data Handling (Ortho Instruments, Westwood, Mass.).

\section{Results}

Human mesothelioma and non-small-cell carcinoma cell lines exhibit differential in vitro sensitivity to the Ad.RSV $t k / G C V$ therapeutic system. To determine the efficacy of the Ad.RSVtk/GCV system on different human thoracic malignant cell types, the in vitro sensitivity to GCV exposure after adenovirus transfer of $\mathrm{HSV} t k$ was evaluated in three mesotheliomas, five non-small-cell carcinomas, and one line each derived from normal thoracic mesothelium and ovarian carcinoma. We also evaluated one rat malignant mesothelioma cell line. Marked differences in GCV dose required for elimination of $50 \%$ of cells as compared with untreated control cells (IC50) was noted (Table I). All me- sothelioma lines exhibited an IC50 GCV dose of less than $3 \mu \mathrm{m}$ (range 0.0075 to $2.8 \mu \mathrm{mol} / \mathrm{L}$ ), whereas the IC50 of the non-small-cell carcinoma cell lines was generally higher, varying from 1.0 to $100 \mu \mathrm{mol} / \mathrm{L}$ GCV. HPM, the cell line derived from normal human mesothelium, required a GCV dose approximately tenfold greater than the least sensitive mesothelioma line and Ovcar-5, a human ovarian carcinoma cell line, was extremely insensitive to the effects of GCV(IC50 > $2000 \mu \mathrm{mol} / \mathrm{L})$.

Expression of the fibronectin-binding receptor $\alpha_{\mathrm{v}}$ does not correlate with $\mathrm{HSV} t \boldsymbol{t} / \mathrm{GCV}$ sensitivity. Although the exact nature of the adenoviral cell surface receptor has not been elucidated, it has recently been shown that interaction with a second set of receptors, the integrins $\alpha_{\mathrm{v}} \beta_{3}$ or $\alpha_{\mathrm{v}} \beta_{5}$, is necessary for internalization of virus. ${ }^{8}$ We therefore determined the density of surface $\alpha_{\mathrm{v}}$ integrin by fluorescence activated cell sorting analysis to see whether the differential ability to express adenovirus-derived proteins might be due to a lack of surface protein needed to internalize adenovirus. As illustrated in Table I and Fig. 2, the amount of $\alpha_{\mathrm{v}}$ expressed by the tumor cells was variable and did not correlate with sensitivity to $\mathrm{HSV} t k / \mathrm{GCV}$ or to $l a c Z$ expression.

Ad.CMVlacZ transduction efficiency correlates with Ad.RSVtk/GCV sensitivity. The resistance of some cell lines to Ad.RSV $t k / G C V$ could be due to many factors including decreased efficiency of viral entry, transcription of transgene, or activity of HSV $t$, as well as an inherent resistance to the effects of phosphorylated GCV products (see Fig. 1). To begin to dissect these mechanisms, we infected the cells with a second adenovirus vector containing the lac $Z$ marker gene under the control of the cytomegalovirus promoter. Failure to express this independent gene would suggest problems with viral entry or internalization rather than resistance to the effects of GCV. As is noted in Table I and Fig. 3, the percentage of Ad.CMVlac $Z$ transduced cells related directly with HSV $t k / G C V$ sensitivity in that it was inversely correlated with the IC50 GCV dose required after Ad.RS$V t k$ infection. The slopes of simple lines fitted to the curves plotting IC50 and percentage of $\beta$-gal expressing cells were similar (13.7 and -12.8 , respectively). Mesothelioma cell lines tended to show a higher relative percentage of lac $Z$ positive cells.

\section{Discussion}

The human mesothelioma cell lines evaluated in this study appeared to be more sensitive to the HSVtk/ 


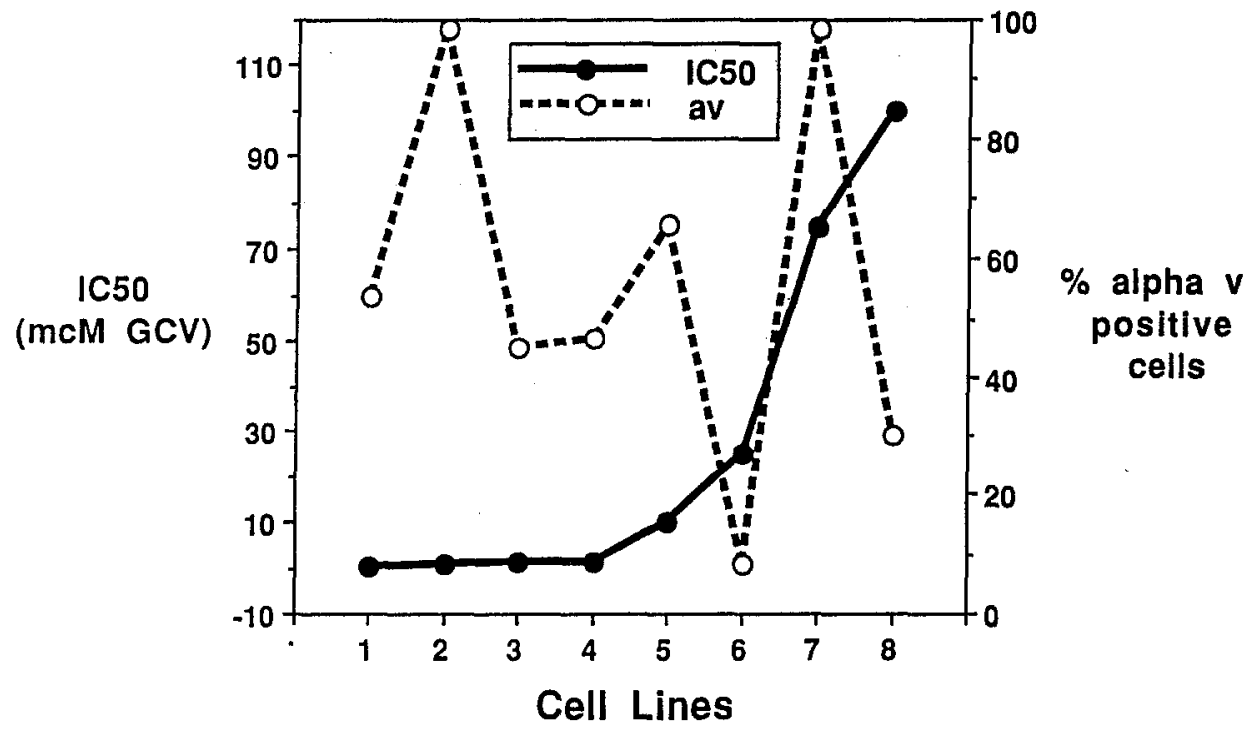

Fig. 2. Comparison of human cell line sensitivity to adenovirus vector-mediated HSVtk system and $\alpha_{\mathrm{v}}$ integrin expression. No obvious relationship exists. Cell line 1, 1-45; line 2, Calu-6; line 3, SK-MES-1; line 4, REN; line 5, H460; line 6, HPM; line 7, A549; line 8, H1264.

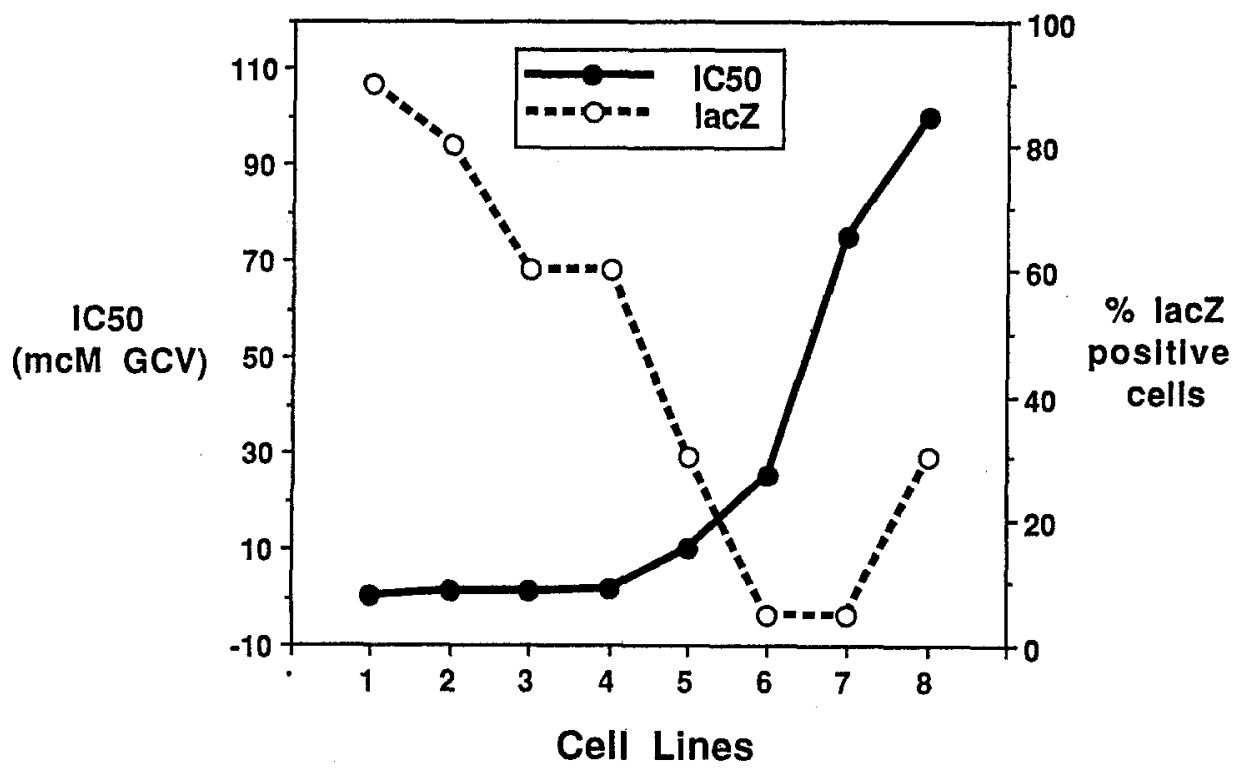

Fig. 3. Comparison of human cell line sensitivity to adenovirus vector-mediated HSV $t k$ system and Ad.CMVlacZ transduction efficiency. The magnitude of simple lines fit to these curves is similar. (See cell line legend to Fig. 2.)

GCV gene therapy system than both non-small-cell carcinoma and normal cell lines tested. The concentration of GCV required to reach IC50 for these mesothelioma cell lines is well below what is routinely achieved in patients $(\sim 20 \mu \mathrm{mol} / \mathrm{L})$ treated for herpesvirus infections at approved clinical doses..$^{14}$ Although the relationship between the sensitivities of cells to
GCV in vitro and in vivo remains to be determined, we have shown that xenograft models of human solid tumor mesothelioma are extremely sensitive to this treatment method in immunocompromised mice, ${ }^{15}$ thus suggesting that the HSV $t k / \mathrm{GCV}$ system may have wider applicability.

As with any new therapy, it is important to analyze 
sensitivity of potential target diseases so that rational decisions may be made regarding indications for use. The data presented here suggest that differences in sensitivity to $\mathrm{HSV} t k / \mathrm{GCV}$ may be due to cellular resistance to adenoviral binding and/or uptake rather than inherent resistance to the effect of the HSVtk enzyme. This conclusion is based on the observation that resistance to GCV is correlated with expression of another adenoviral vector containing a marker gene, Ad.CMVlacZ. Although more work will be required to determine which step in the viral cycle (see Fig. 1) is blocked in resistant cells, our study does suggest that the problem is not due simply to lack of expression of vitronectin receptor by resistant cell lines. Evaluation of initial viral binding to adenoviral cell surface receptors, using radiolabeled virus, ${ }^{9}$ may be useful in resolving this issue further.

In summary, this study has shown that sensitivity to the $\mathrm{HSV} t k / \mathrm{GCV}$ can vary over a wide range. Mesothelioma and non-small-cell lung cancer cell lines are quite sensitive, whereas at least one ovarian carcinoma line was extremely resistant. The mechanism for this resistance is unknown but appears to be due to factors related to viral uptake, internalization, or expression rather than resistance to the HSVtk enzyme. An understanding of the relative sensitivities of various neoplasms to this gene therapy technique and the mechanisms responsible for this sensitivity will lead to rational and targeted clinical application.

\section{REFERENCES}

1. Rusch VW, Pintadosi S, Holmes EC. The role of extrapleural pneumonectomy in malignant mesothelioma. J THORAC CARdIOVASC SURG 1991;102:1-9.

2. Sugarbaker DJ, Heher EC, Lee TH, et al. Extrapleural pneumonectomy, chemotherapy, and radiotherapy in the treatment of diffuse malignant mesothelioma. $J$ THORAC CARdiovasc SURg 1991;102:10-15.

3. Smythe WR, Kaiser LR, Hwang HC, et al. Successful adenovirus-mediated gene transfer in an in vivo model of human malignant mesothelioma. Ann Thorac Surg [In press].

4. Smythe WR, Hwang HC, Amin KM, et al. Use of recombinant adenovirus to transfer the herpes simples thymidine kinase (HSVtk) gene to thoracic neoplasms: an effective in vitro drug sensitization system. Cancer Res 1994;54:2055-9.

5. Matthews T, Boehme R. Antiviral activity and mechanism of action of ganciclovir. Rev Inf Dis 1988;10:s490-4.

6. Freeman SM, Abboud CN, Whartenby KA, et al. The "bystander effect": tumor regression when a fraction of the tumor mass is genetically modified. Cancer Res 1993;53:5274-83.

7. Li Bi W, Parysek LM, Warnick R, Stambrook PJ. In vitro evidence that metabolic cooperation is responsible for the bystander effect observed with HSVtk retroviral gene therapy. Hum Gene Ther 1993;4:725-31.

8. Wickham TJ, Mathias P, Cheresh DA, Nemerow GR. Integrins $\alpha_{v} \beta_{3}$ and $\alpha_{v} \beta_{5}$ promote adenovirus internalization but not attachment. Cell 1993;73:309-19.

9. Greber UF, Willets M, Webster P, Helenius A. Stepwise dismantling of adenovirus 2 during entry into cells. Cell 1993;75:477-86.

10. Englehardt JF, Yang Y, Stratfor-Perricaudet LD, et al. Direct gene transfer of human CFTR into human bronchial epithelia of xenografts with E1-delated adenoviruses. Nat Genet 1993;4:27-34.

11. Berkner KL. Development of adenovirus vectors for the expression of heterologous genes. Biotechniques 1988;6:616-29.

12. Brunner N, Thompson EW, Spang-Thomsen M, Rygaard J, Dano K, Zweibel JA. lacZ transduced human breast cancer xenografts as an in vivo model for the study of invasion and metastasis. Eur J Cancer 1992; 28A:1989-1995.

13. Cheresh D, Harper J. Arg-Gly-Asp recognition by a cell adhesion receptor requires its $130 \mathrm{kDa} \alpha$ subunit. J Biol Chem 1987;262:17703-11.

14. Paul S, Drummer S. Topics in clinical pharmacology: ganciclovir. Am J Med Sci 1992;304:272-7.

15. Smythe WR, Hwang HC, Amin KM, et al. Treatment of experimental human mesothelioma using adenovirus transfer of the herpes simplex thymidine kinase gene. Ann Surg [In press].

\section{Discussion}

Dr. Martin F. McKneally (Toronto, Ontario, Canada). Can you tell us anything about your hopes in terms of application in the human model? Will it be limited only to the tumor cell, or if there is spillover to the normal cells, will it be destructive or harmful?

Dr. Smythe. We and others have shown that when recombinant adenovirus is placed into a body cavity lined with mesothelium the normal mesothelium is infected along with any tumor that may be growing in that cavity; however, it seems to form a barrier to penetration of the virus into normal tissues. It does not easily traverse the normal mesothelium.

In addition, it appears that normal cells that are not rapidly dividing and are not rapidly synthesizing deoxyribonucleic acid (DNA) and transcribing as much DNA as tumor cells are not affected to the same degree.

We picked mesothelioma as a model system because we thought it was an ideal tumor, basically because most morbidity is from local disease, and its anatomic distribution may allow us to treat without as much concern about generalized toxicity.

I would like to add that we have actually treated intraperitoneal preexisting human tumor in our immunedeficient mouse model. In contrast to the work I described here, in which cells are infected in vitro, in these experiments we infected tumor after it had already been established in the peritoneal cavity, much as you would see in 
the clinical situation. We found that in $90 \%$ of our animals we completely eliminated macroscopic disease, and we also found that in $80 \%$ of animals we completely eradicated microscopic disease as well. We are currently working on in vivo preclinical toxicity studies with hopes of submitting this protocol to the DNA Recombinant Advisory Committee this fall for consideration of a possible clinical trial.

Dr. Pedro J. del Nido (Pittsburgh, Pa.). You are using adenovirus, which sometimes can have variable expression, as a vector. Have you considered just the adeno-associated viruses, with which some people have had better expression?

Dr. Smythe, No, actually the adenovirus to date, in a number of studies comparing head to head all methods of gene transfer, is by far the most effective and efficient method, much more effective than retrovirus or conventional methods.

The adeno-associated virus is a different virus. It is a virus that requires co-infection with adenovirus to engender gene transfer. There are significant technical problems right now with production of sufficient amounts of adenoassociated virus, and it appears to be a relatively inefficient gene transfer vector.

Dr. Donald L. Morton (Santa Monica, Calif.). How long will this transfer gene persist in vitro or in vivo? Do the cells eliminate the transfer gene or do they continue to express it for a long period?

Dr. Smythe. Adenoviral gene transfer is transient compared with some other methods. A trade-off is efficiency of gene transfer. In the case of correction of genetic diseases such as cystic fibrosis and familial hypocholesterolemia, it would be important to have prolonged expression of a gene. For transfer of a toxic or suicide gene, as in this study, we believe that durable expression is not warranted. Our goal is quick eradication of the tumor.

In addition, there does seem to be an immune response to adenovirus. Most of us have seen adenovirus at some point in our lives. However, in some studies this has been shown to be beneficial in treatment of malignancy. The immune response is actually additive to the toxic effect of the gene.

Dr. Morton. If the gene does not persist, you theoretically would have the same problem that you have with chemotherapy: resistance. The cells that develop resistance will be the ones that regrow.

Dr. Smythe. I do not know that resistance to these sorts of therapies has ever been shown in vitro, at least for the transfer of toxic or suicide genes. The genes do persist for at least 3 weeks even in immune competent animals, which would allow a fairly prolonged treatment time, equivalent to what many chemotherapy regimens are. 